\title{
Métodos para Avaliação da Exposição a Poluentes Plásticos em Procellariiformes: Revisão e Padronização de Protocolos
}

\author{
Luciana Gallo ${ }^{1}$, Marcela Uhart ${ }^{2}$, Alice Pereira ${ }^{3}$ \& Patricia Pereira Serafini ${ }^{4}$
}

Recebido em 02/02/2021 - Aceito em 16/09/2021

1 Instituto de Biología de Organismos Marinos/IBIOMAR, Consejo Nacional de Investigaciones Científicas y Técnicas/CONICET, Puerto Madryn/Chubut, Argentina. < gallo@cenpat-conicet.gob.ar>.

2 Latin America Program, Karen C. Drayer Wildlife Health Center/WHC, School of Veterinary Medicine, University of California/UC, Davis/ California, USA. <muhart@ucdavis.edu>.

3 Projeto Albatroz, Florianópolis/SC, Brasil. < apereira@projetoalbatroz.org.br>.

4 Instituto Chico Mendes de Conservação da Biodiversidade/ICMBio, Centro Nacional de Pesquisa e Conservação de Aves Silvestres/CEMAVE, Florianópolis/SC, Brasil. CEP: 88.053-700. <patricia.serafini@icmbio.gov.br>.

RESUMO - A presença de resíduos antropogênicos em águas oceânicas e sua ingestão por aves marinhas tem sido alvo de um crescente número de estudos. Os Procellariiformes são particularmente suscetíveis à ingestão de plástico, uma vez que se alimentam preferencialmente de pequenas presas na superfície da água, onde os plásticos tendem a flutuar e se acumular. Após revisão bibliográfica e aplicação prática de técnicas em campo e laboratório, apresentamos um protocolo padronizado de amostragem para a avaliação da ingestão de plásticos por Procellariiformes que inclui recomendações para opções de tipos e fontes de amostras, além de adaptações à coleta para atender a diversos objetivos de pesquisa. As amostras podem ser coletadas de animais mortos oriundos da captura incidental em atividades de pesca; encalhes de praia; aves mortas nas colônias ou centros de reabilitação; animais vivos em colônias ou centros de reabilitação; ou amostragem não invasiva por meio das fezes, bolos alimentares e ovos não eclodidos. Além disso, sugerimos tipos de análises possíveis, materiais necessários e rotinas de limpeza para evitar a contaminação durante a coleta e processamento. $\mathrm{O}$ uso de protocolos padronizados aumenta a consistência, comparabilidade e a reprodutibilidade, permitindo comparações entre estudos em escalas temporais e espaciais diferenciadas.

Palavras-chave: Oceano; poluição; aves marinhas; resíduos plásticos.

\section{Methods for Assessing Exposure to Plastic Pollutants in Procellariiformes: Review and Standardization of Protocols}

\begin{abstract}
The presence of anthropogenic debris in oceanic waters and their ingestion by seabirds has been the subject of a growing number of studies. Procellariiformes are particularly susceptible to plastic ingestion, since they feed preferably on small prey on the waters' surface, where plastics tend to float and accumulate. Following an extensive literature review and practical application of techniques in the field and laboratory by the authors, we present a set of guidelines for sampling Procellariiformes to assess plastic ingestion. The guidelines suggest several sample type and sample source options, offering ways to approach different research objectives and overcome logistics constraints. Samples may be collected from dead beach-cast birds or those caught incidentally by fisheries. It is also possible to collect samples from live or dead animals in their breeding sites, rehabilitation centers, or noninvasively through feces, boluses and non-hatched eggs. In addition, we recommend analysis methods, necessary materials and cleaning routines to avoid contamination during collection and processing. The use of standardized protocols increases consistency and repeatability, allowing comparisons between investigations for a number of species, as well as the detection of large-scale spatiotemporal patterns.
\end{abstract}

Keywords: Ocean; plastic debris; pollution; seabirds. 


\title{
Métodos para Evaluar la Exposición a Contaminantes Plásticos en Procellariiformes: Revisión y Estandarización de Protocolos
}

\begin{abstract}
RESUMEN - Los estudios sobre la presencia de residuos antropogénicos en el mar y su ingesta por aves marinas se han incrementado en las últimas décadas. Los Procellariiformes son particularmente susceptibles a la ingesta de plásticos debido a que se alimentan preferentemente de presas sobre la superficie del mar, donde los plásticos tienden a flotar y acumularse. Luego de una extensa revisión bibliográfica y la aplicación práctica de técnicas en el laboratorio y el campo, presentamos protocolos estandarizados para la colecta de muestras para evaluar la ingesta de plásticos en Procellariiformes. Estos protocolos sugieren una variedad de tipos y fuentes de muestras y metodologías de colecta de acuerdo a diferentes objetivos de investigación. Las muestras pueden ser colectadas de aves muertas encontradas en la playa o capturadas incidentalmente en pesquerías; de aves vivas o muertas en colonias reproductivas y centros de rehabilitación; o de manera no-invasiva en el caso de fecas, bolos y huevos no eclosionados. Además, recomendamos métodos de análisis, insumos necesarios y rutinas de limpieza para evitar la contaminación durante la colecta y procesamiento de muestras. La implementación de protocolos estandarizados para la colecta y el análisis de muestras favorece la consistencia y repetibilidad de los estudios, permitiendo la comparación de los resultados y la detección de patrones espacio-temporales a gran escala.
\end{abstract}

Palabras clave: Océano; polución; aves marinas; residuos plásticos.

\section{Introdução}

Albatrozes e petréis (Procellariiformes) estão entre as espécies de aves marinhas mais ameaçadas de extinção, com populações diminuindo rapidamente e seu estado de conservação deteriorando-se acentuadamente nos últimos anos (Birdlife International, 2018; Phillips et al., 2016). Ainda que a captura incidental em atividades de pesca seja a mais importante causa de mortalidade e declínio populacional para essas aves desde o final dos anos 1980, outros fatores como a introdução de espécies exóticas invasoras e os patógenos nas colônias, a poluição marinha $e$ a ingestão de resíduos de origem antropogênica foram reconhecidas como ameaças para suas populações (Phillips et al., 2016; Dias et al., 2019).

Procellariiformes são aves particularmente suscetíveis à ingestão de plástico, pois se alimentam preferencialmente de presas pequenas em águas superficiais, sendo que os resíduos plásticos tendem a boiar exatamente nessa mesma coluna de água (Titmus \& Hyrenbach, 2011; Sebille et al., 2020). Além disso, estudos recentes mostraram que o biofilme que se forma na superfície dos resíduos plásticos produz uma assinatura olfativa que se assemelha à do alimento natural de Procellariiformes, o que também explicaria a alta frequência de ingestão de resíduos nesse grupo (Savoca et al., 2016). A quantidade crescente de resíduos plásticos circulando nos oceanos (Jambeck et al., 2015), a crescente evidência de sua ingestão intencional e acidental pelas aves marinhas (Wilcox et al., 2015) e a lacuna de conhecimento sobre os efeitos (individuais e/ou populacionais) de sua ingestão evidenciam a importância de priorizar sua investigação (Weimerskirch et al., 2003; Parsons et al., 2008; Grimaldi et al., 2015; Phillips et al., 2016).

O conhecimento atual sobre os efeitos negativos dos resíduos plásticos na vida silvestre é amplamente baseado em consequências facilmente observadas, como emalhamento, obstrução do intestino ou caquexia (Pierce et al., 2004; Phillips et al., 2010; Ryan et al., 2016; Roman et al., 2019). Muitas dessas interações também incluem efeitos subletais menos visíveis, até o momento pouco documentados (Rochman et al., 2014; Limonta et al., 2019; Fossi et al., 2020). Ainda, a acumulação de compostos químicos derivados da degradação dos resíduos plásticos e aditivos como plastificantes (ftalatos) e retardantes de chama (éteres difenílicos polibromados - PBDE) já é documentada em aves marinhas (Tanaka et al., 2013; 2015; Hardesty et al., 2015; Tanaka et al., 2020). Muitos desses compostos são potencialmente tóxicos e são conhecidos por induzir uma variedade de efeitos crônicos e subletais, incluindo disfunção endócrina, neurotoxicidade, distúrbios metabólicos, disrupção de resposta do sistema imune, mutagênese e carcinogênese (Prokic et al., 2019; Puskic et al., 2020). A acumulação desses compostos (e.g. a partir de lixiviação crônica de partículas de plástico retidas no estômago) pode 
afetar o ciclo de vida e o sucesso reprodutivo das espécies, potencialmente contribuindo com redução populacional em longo prazo (Finkelstein et al., 2007; Hardesty et al., 2015).

Além dos compostos derivados da degradação dos resíduos plásticos, compostos tóxicos como bifenilpoliclorados (PCBs) e os pesticidas organoclorados (POCs) possuem afinidade por partículas poliméricas como o plástico e tendem a serem adsorvidos pelas mesmas (Mato et al., 2001; Endo et al., 2005; Ríos et al., 2007). A adsorção é um fenômeno pelo qual compostos orgânicos hidrófobos se concentram e se aderem na superfície do plástico (Mato et al., 2001; Endo et al., 2005; Provencher et al., 2018a). A presença desses compostos foi detectada em fragmentos de plástico ingeridos por aves marinhas no Atlântico Sudoeste (Colabuono et al., 2010).

Para investigar a ingestão de plástico nas aves marinhas é importante utilizar uma metodologia padrão (van Franeker et al., 2011). O desenvolvimento de métodos para a amostragem $e$ análise dos resíduos plásticos é uma área de pesquisa importante e emergente (Provencher et al., 2019; Kühn \& van Franeker, 2020). Atualmente, Fulmarus glacialis é a única espécie para a qual um protocolo padrão de coleta $e$ análise foi adotado para determinar a ingestão de resíduos plásticos (itens $>1 \mathrm{~mm}$ ) através de análise de conteúdo estomacal (OSPAR, 2015). Esses métodos foram aplicados com sucesso em estudos no Atlântico e Pacífico Norte, permitindo comparações entre espécies e ao longo do tempo e do espaço (van Franeker et al., 2011; Provencher et al., 2017; Avery-Gomm et al., 2018; Provencher et al., 2019).

No presente trabalho apresentamos um protocolo padronizado de amostragem de resíduos plásticos para coletas de albatrozes e petréis vivos ou mortos e/ou ambiental, de acordo com diferentes objetivos de pesquisa. Além disso, sugerimos tipos de análises possíveis, materiais necessários e rotinas de limpeza para evitar a contaminação durante a coleta e processamento $\mathrm{de}$ amostras. Os protocolos aqui apresentados podem ser aplicados amplamente tanto em campo por uma equipe inexperiente (e.g. amostragem ambiental $e$ de aves mortas), bem como por pessoal especializado no caso de aves vivas ou equipes executando necropsias completas em condições controladas. Embora o foco deste trabalho seja dado a Procellariiformes, essas diretrizes e recomendações são generalizáveis para outras espécies de aves marinhas e outros grupos taxonômicos.

\section{Material e Métodos}

Para a pesquisa bibliográfica foram usadas as plataformas e bases de dados Google Scholar, SpringerLink, ScienceDirect, Taylor and Francis Online, Wiley Online Library na busca de artigos científicos peer-reviewed, teses e dissertações. As palavras-chave incluíram "plastic ingestion", "plastic debris", "plastic additives", "Procellariiformes", "seabirds", "sampling protocol" e "sample procedure". Estudos publicados durante as últimas duas décadas em aves marinhas foram considerados.

Embora o enfoque taxonômico das buscas tenha sido dado para Procellariiformes e aves marinhas, também foram considerados estudos para outros grupos taxonômicos que pudessem ser extrapolados para a Classe Aves. Informação sobre o tipo de estudo, metodologia de coleta para os diferentes tipos de amostras, processamento e análise laboratorial foram extraídos de cada referência.

Também foram consideradas na revisão os comentários e recomendações dos protocolos apresentados pelas autoras deste trabalho ao Acordo para a Conservação de Albatrozes e Petréis (ACAP) durante a $11^{\mathrm{a}}$ reunião do Comitê Consultivo em 2019, que foram consolidados em guia de boas práticas do ACAP (Uhart et al., 2020), e a aplicação prática posterior dos procedimentos em campo por meio de projeto financiado por este Acordo.

Neste trabalho, são tratados como macroplásticos os resíduos $>5 \mathrm{~mm}$ e microplásticos aqueles $<5 \mathrm{~mm}$ conforme GESAMP (2019).

\section{Resultados e Discussão}

\section{Revisão bibliográfica e tipos de análises possíveis}

Foram identificadas 70 referências para o desenvolvimento de protocolos padronizados para investigar a exposição das aves marinhas ao plástico. Na Figura 1 apresentamos o número 
de artigos por ano de publicação. Somente 10 (7\%) destas publicações correspondem a estudos desenvolvidos no Oceano Atlântico Sudoeste, principalmente com Procellariiformes (Copello \&
Quintana, 2003; Copello et al., 2008; Colabuono et al., 2009, 2010; Tourinho et al., 2010; Jiménez et al., 2015; Lenzi et al., 2016; Tavares et al., 2017; Petry \& Benemann, 2017; Yorio et al., 2020).

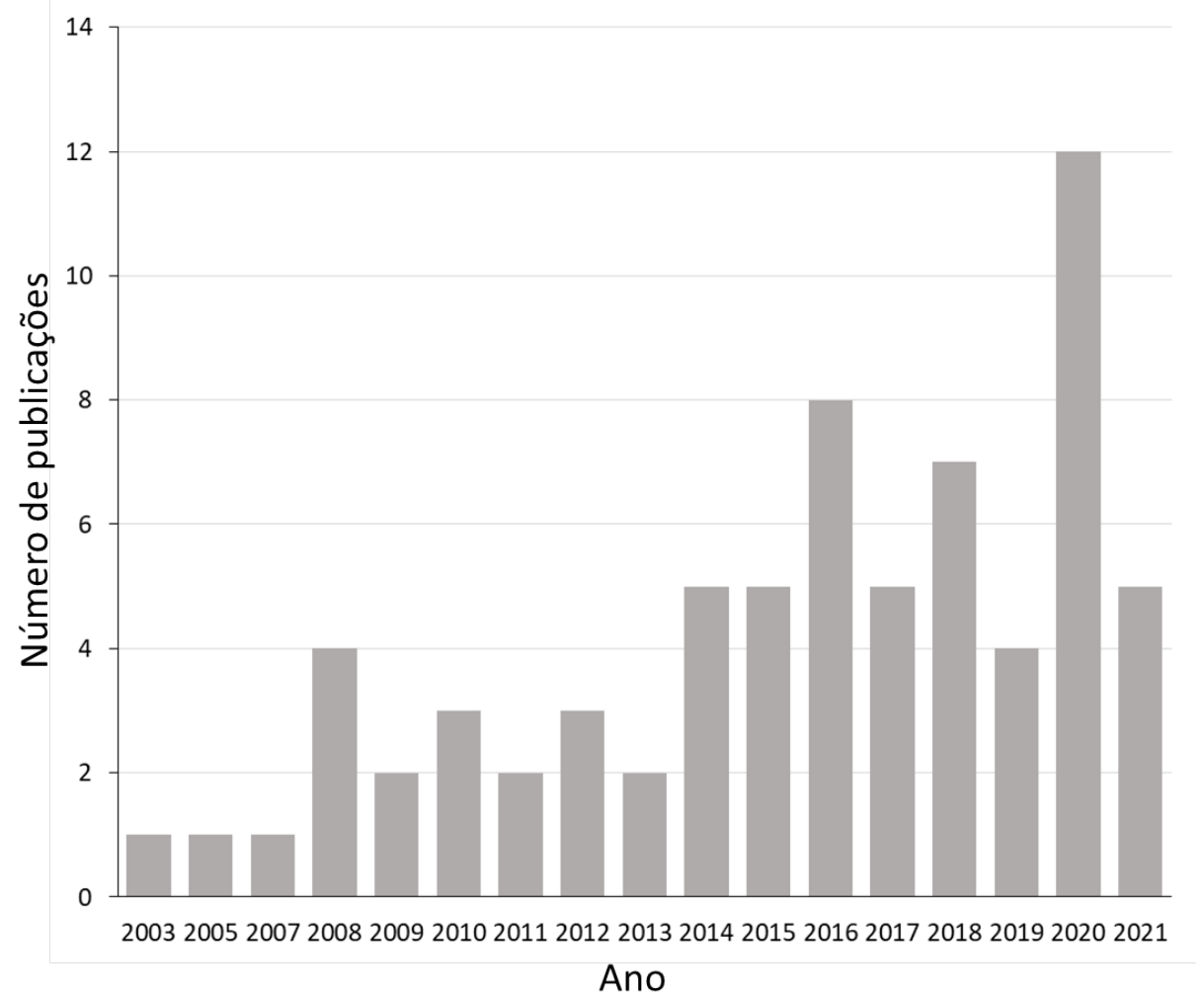

Figura 1 - Visualização em escala temporal do número de publicações consultadas para a elaboração dos protocolos apresentados no presente artigo.

$\mathrm{Na}$ revisão das publicações disponíveis até a presente data, em um nível crescente de complexidade, observa-se que é possível executar ao menos três níveis potenciais de análises (Figura 2): 1) detecção, triagem e quantificação de itens plásticos em conteúdo gastrointestinal, bolos alimentares ou fezes; 2) caracterização dos itens triados; e 3) análise química de compostos associados aos plásticos em ovos não viáveis ou não eclodidos, óleo estomacal, glândula uropigial, órgãos e tecidos (compostos aditivos), e itens triados (compostos aditivos e adsorvíveis).

Na Tabela 1 apresentamos toda a bibliografia consultada para a elaboração dos protocolos presentes neste artigo, de acordo com os tipos de análises pretendidas, amostras e fontes. A partir deste levantamento geral, são aprofundados os materiais e os protocolos de coleta de amostras recomendados (Tabela 2 ).

Tabela 1 - Bibliografia consultada para a elaboração de protocolos para coleta de amostras tendo em conta análise, fonte e tipos de amostra.

\begin{tabular}{|c|c|}
\hline \multicolumn{2}{|r|}{ Detecção, triagem e quantificação de itens plásticos } \\
\hline \multicolumn{2}{|l|}{ Aves vivas } \\
\hline conteúdo gastrointestinal & $\begin{array}{l}\text { Carey, 2011; Lavers et al., 2014; Provencher et al., 2014; Copello \& Quintana, 2003; Copello et al., } 2008 \text {; } \\
\text { Phillips et al., 2010, 2020; Yorio et al., } 2020 .\end{array}$ \\
\hline \multicolumn{2}{|l|}{ Aves mortas } \\
\hline conteúdo gastrointestinal & $\begin{array}{l}\text { Mallory et al., 2008; Colabuono et al., 2009; Tourinho et al., 2010; Gray et al., 2012; Codina-Garcia et al., } \\
\text { 2013; Bond et al., 2014; Jimenez et al., 2015; Acampora et al., 2016; Herzke et al., 2016; Poon et al., 2017; } \\
\text { Petry \& Benemann, 2017; Ryan, 2008, 2016; Roman et al., 2016, 2019; Hyrenbach et al., 2017; Provencher } \\
\text { et al., 2009, 2014, 2018b, van Franeker et al., 2011; Tavares et al., 2017; Avery-Gomm et al., 2018; Lavers } \\
\text { et al., 2019; Avery-Gomm, 2020; Baak et al., 2020; Padula et al., 2020; Kühn, 2020; Bourdages et al., 2021; } \\
\text { Hamilton et al., 2021; Kühn et al., 2021. }\end{array}$ \\
\hline
\end{tabular}




\begin{tabular}{|c|c|}
\hline \multicolumn{2}{|l|}{ Ambiente } \\
\hline bolos alimentares & $\begin{array}{l}\text { Lindborg et al., 2012; Hammer et al., 2016; Lenzi et al., 2016; Hyrenbach et al., 2017; Perold et al., 2020; } \\
\text { Stewart et al., 2020; Ibañez et al., 2020; Yorio et al., 2020; Bond et al., } 2021 .\end{array}$ \\
\hline fezes & Gil-Delgado et al., 2017; Le Guen et al., 2020. \\
\hline \multicolumn{2}{|r|}{ Detecção, triagem e quantificação de itens plásticos e Caracterização dos itens triados } \\
\hline \multicolumn{2}{|l|}{ Aves vivas } \\
\hline conteúdo gastrointestinal & $\begin{array}{l}\text { Lavers et al., 2014; Provencher et al., 2014; Copello \& Quintana 2003; Copello et al., 2008; Phillips et al., } \\
\text { 2010, 2020; Yorio et al., 2020; Lusher et al., } 2018 .\end{array}$ \\
\hline \multicolumn{2}{|l|}{ Aves mortas } \\
\hline \multirow[t]{2}{*}{ conteúdo gastrointestinal } & $\begin{array}{l}\text { Mallory et al., 2008; Provencher et al., 2009; Gray et al., 2012; Codina-Garcia et al., 2013; Bond et al., 2014; } \\
\text { Acampora et al., 2016; Avery-Gomm et al., 2016, 2020; Tavares et al., 2017; Baak et al., 2020; Yorio et al., } \\
\text { 2020; Kühn, 2020; Bourdages et al., 2021; Hamilton et al., 2021; Kühn et al., } 2021 .\end{array}$ \\
\hline & Lusher et al., 2015, 2018 (mamíferos marinhos). \\
\hline \multicolumn{2}{|l|}{ Ambiente } \\
\hline bolos alimentares & $\begin{array}{l}\text { Nilsen et al., 2014; Lenzi et al., 2016; Álvarez et al., 2018; Perold et al., 2020; Ibañez et al., 2020; Stewart et } \\
\text { al., 2020; Yorio et al., 2020; Bond et al., } 2021 .\end{array}$ \\
\hline \multirow[t]{2}{*}{ fezes } & Le Guen et al., 2020. \\
\hline & Lusher et al., 2018; Nelms et al., 2019 (mamíferos marinhos) \\
\hline \multicolumn{2}{|r|}{ Análises químicas } \\
\hline \multicolumn{2}{|l|}{ Compostos aditivos } \\
\hline \multicolumn{2}{|l|}{ Aves vivas } \\
\hline óleo estomacal & Tanaka et al. 2015. \\
\hline óleo da glândula uropigial & Hardesty et al., 2015. \\
\hline \multicolumn{2}{|l|}{ Aves mortas } \\
\hline óleo estomacal & Tanaka et al., 2015; Kühn, 2020; Kühn et al., 2020. \\
\hline $\begin{array}{l}\text { itens plásticos de conteúdo } \\
\text { estomacal }\end{array}$ & Tanaka et al., 2013, 2015, 2018, 2019; Herzke et al., 2016; Neumann et al., 2021. \\
\hline \multirow{2}{*}{$\begin{array}{l}\text { tecido adiposo, abdominal } \\
\text { e músculo peitoral, órgãos } \\
\text { (fígado) }\end{array}$} & $\begin{array}{l}\text { Tanaka et al., 2013, 2015, 2020; Herzke et al., 2016; Commendatore et al., 2018; Padula et al., 2020; } \\
\text { Neumann et al., 2021. }\end{array}$ \\
\hline & Fossi et al., 2012, 2014; Baini et al., 2017 (mamíferos marinhos). \\
\hline óleo da glândula uropigial & Hardesty et al., 2015; Tanaka et al., 2020. \\
\hline \multicolumn{2}{|l|}{ Ambiente } \\
\hline $\begin{array}{l}\text { ovos não-viáveis ou não- } \\
\text { eclodidos }\end{array}$ & Jaspers et al., 2005; Polder et al., 2008; Braune et al., 2015; Commendatore et al., 2018. \\
\hline bolos alimentares & Tanaka et al., 2019. \\
\hline \multicolumn{2}{|c|}{ Compostos adsorvíveis em itens plásticos } \\
\hline \multicolumn{2}{|l|}{ Aves vivas } \\
\hline conteúdo estomacal & Ríos et al., 2007. \\
\hline \multicolumn{2}{|l|}{ Aves mortas } \\
\hline conteúdo estomacal & Colabuono et al., 2010; Yamashita et al., 2011; Herzke et al., 2016; Provencher et al., 2018a. \\
\hline \multicolumn{2}{|l|}{ Ambiente } \\
\hline bolos alimentares & Ríos et al., 2007; Tanaka et al., 2019. \\
\hline
\end{tabular}

Os métodos analíticos usados para detecção e triagem de itens plásticos são diversos e dependem do tamanho dos itens plásticos analisados, do tipo de amostra e da quantidade de material orgânico na mesma. No entanto, podem ser agrupados em três subcategorias: (a) exame visual a olho nu (Avery-Gomm, 2020); (b) exame visual com microscópio óptico (com ou sem coloração fluorescente seletiva utilizando vermelho do Nilo) (e.g. Avery-Gomm et al., 2018; Padula et al., 2020); e (c) digestão da amostra com subsequente filtração $e$ análise visual (usualmente utilizado para itens menores a $1 \mathrm{~mm}$ e/ou amostras com elevado conteúdo de matéria orgânica) (e.g. Provencher 
et al., 2018b; Lavers et al., 2019; Hamilton et al., 2021).

Outro conjunto de análises possíveis inclui a caracterização dos itens triados: (a) física (tamanho, forma, tipo, cor) de acordo com os códigos estabelecidos por United Nations Joint Group of Scientific Experts on Scientific Aspects of Marine Environmental Protection (GESAMP, 2019) (e.g. van Franeker et al., 2011; Provencher et al., 2017); (b) química, de acordo com os códigos utilizados pela Society of Plastics Industry (Nilsen et al., 2014) e por espectroscopia (e.g. Lusher et al., 2015, 2018; Avery-Gomm et al., 2016, 2020; Álvarez et al., 2018; Nelms et al., 2019). A espectroscopia de infravermelho transformada de Fourier (espectroscopia FTIR) e Raman, são aplicadas para verificar se os itens suspeitos são polímeros sintéticos, para determinar a composição geral dos itens isolados, ou ambos.

Por último, encontram-se análises químicas para identificar e quantificar compostos associados aos plásticos (aditivos e adsorvíveis) através do uso de técnicas envolvendo cromatografia gasosa e outros métodos de detecção de componentes químicos (e.g. Hardesty et al., 2015; Tanaka et al., 2020). Este conjunto de análises engloba procedimentos que requerem a implementação de medidas de limpeza específicas e a coleta de amostras de controle, também denominadas brancos. Brancos tratam da obtenção de amostras de controle negativo para verificar a existência de contaminação cruzada por partículas de plástico procedente de outras fontes durante a coleta $e$ processamento de amostras (Tanaka et al., 2020).

Como primeiro passo para tomar qualquer decisão em relação ao protocolo a ser escolhido, deve-se selecionar o tipo de análise de acordo com a pergunta-chave a ser respondida, bem como a disponibilidade de recursos e equipamentos, além dos objetivos da pesquisa. Propõe-se um fluxo de pesquisa com diferentes alternativas de análises $e$ tipos de amostras de acordo a perguntas-chave associadas os objetivos de pesquisa (Figura 2). Os protocolos de coleta e armazenamento de amostras podem ser consultados na Tabela 2 .

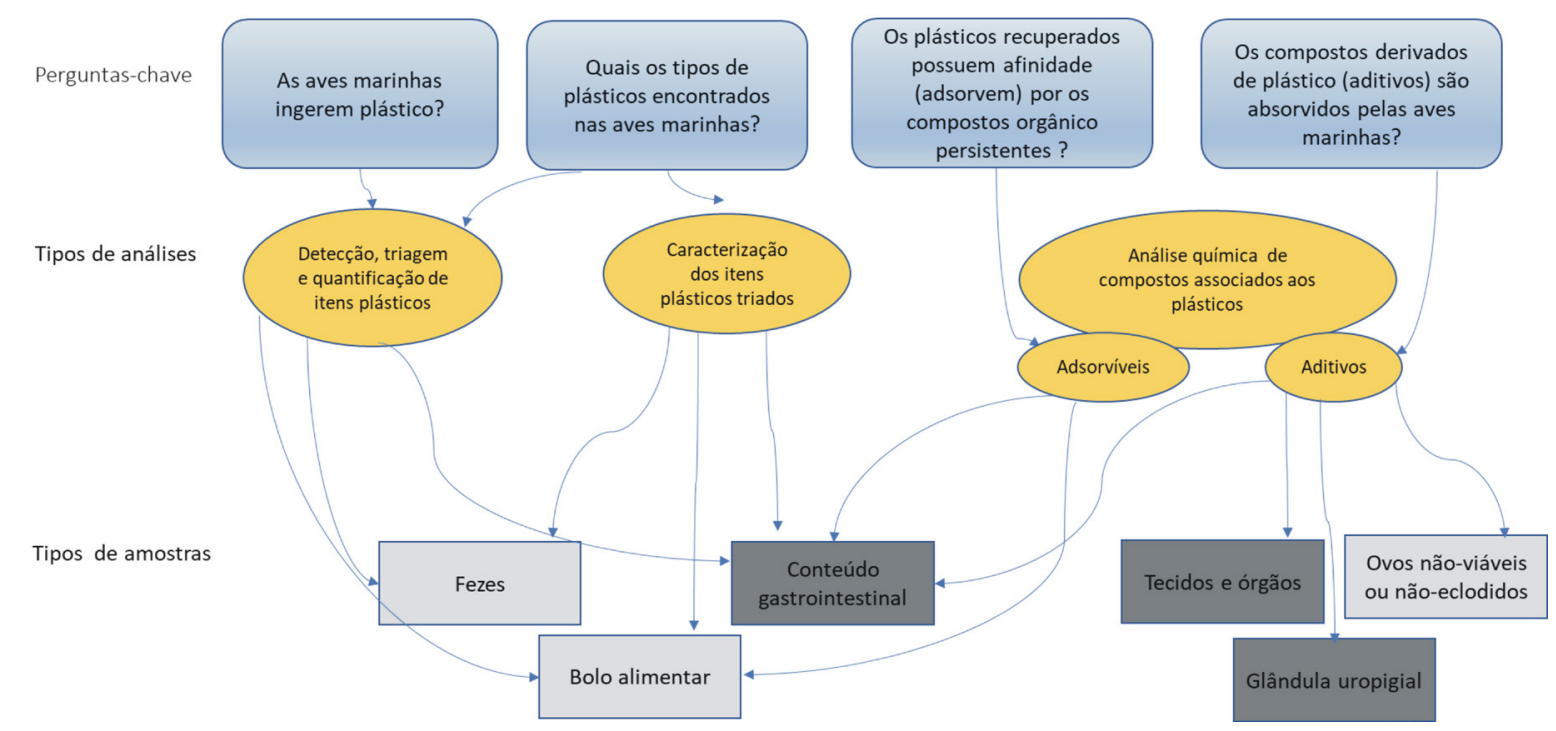

Figura 2 - Fluxo de pesquisa para a avaliação de ingestão de plásticos com diferentes alternativas de análises e tipos de amostras de acordo a perguntas-chave associadas os objetivos de pesquisa. Fonte de amostra: cinza escuro (ave viva ou morta), cinza claro (ambiental).

\section{Protocolos padronizados de coleta $e$ armazenamento de amostras}

As referências consultadas utilizam-se de amostras coletadas de aves mortas (59\%), vivas (9\%), ou ambas (4\%). Entretanto, nos últimos anos, observa-se um aumento de trabalhos que se utilizam de amostragem não invasiva por meio da coleta de fezes, bolos alimentares e ovos, tanto como única fonte (21\%) ou em combinação com amostras de aves vivas e/ou mortas (7\%). A Tabela 2 apresenta as considerações metodológicas padronizadas para a coleta $e$ armazenamento de amostras de acordo com sua 
fonte e seu tipo, bem como de acordo com a análise a ser realizada.

Aves mortas (e.g. oriundas da captura incidental, encalhes de praia, mortas nas colônias ou centros de reabilitação): a coleta pode ser executada em carcaças recém obtidas ou armazenadas congeladas. A necropsia do espécime deve ser realizada, preferencialmente, dentro de um ambiente controlado (e.g. laboratório) para reduzir o risco de contaminação. A contaminação oriunda do ambiente é especialmente prejudicial para futura análise de poluentes nas amostras coletadas e/ou investigação da presença de itens menores que $1 \mathrm{~mm}$. As carcaças frescas não são requisito obrigatório para a análise, contudo, quando há predação por carniceiros deve-se notar se houve dano ou retirada do trato digestório. $\mathrm{O}$ trato gastrointestinal (esôfago, pro-ventrículo, ventrículo $e$ intestino), órgãos, tecidos e glândula uropigial podem ser coletados por meio da dissecação ou durante a necropsia (detalhes da coleta na Tabela 2).

Aves vivas (e.g. em centros de reabilitação ou colônias): detalhes da coleta das amostras (e.g. conteúdo estomacal e óleo de glândula uropigial) são apresentados na Tabela 2. O trabalho com aves vivas requer treinamento específico $e$ deve estar restrito a pessoas com experiência para a atividade. O conteúdo estomacal (sólido e oleoso) pode ser coletado por regurgitação espontânea voluntária (Phillips, 2006; Provencher et al., 2014; McInnes et al., 2016). A lavagem estomacal (ou emética) é altamente invasiva e não recomendada para esse propósito. A regurgitação forçada pode causar danos e mortalidade, além de incutir viés às amostras obtidas (Provencher et al., 2019). Além disso, o ventrículo dos Procellariiformes (exceto o dos albatrozes) é separado do proventrículo por um istmo no qual os itens rígidos podem permanecer alojados e, portanto, não serão facilmente regurgitados (Furness et al., 1985). Por estes motivos, as técnicas envolvendo a regurgitação raramente rendem uma amostra completa (Provencher et al., 2019).

Ambiental: Detalhes sobre a coleta de amostras ambientais podem ser consultados na Tabela 2. Fezes frescas, bolos alimentares do ninho e das imediações, e ovos (não viáveis ou não eclodidos) são amostras úteis e não invasivas para a determinação da ingestão de resíduos plásticos (itens $\leq 5 \mathrm{~mm} e>1 \mathrm{~mm}$ ) $e$ contaminantes associados, e que não requerem a manipulação das aves. Bolos alimentares são materiais não digeríveis contendo itens alimentares naturais, mas também podem conter resíduos de origem antropogênica. Bolos são regurgitados espontaneamente por adultos ou ninhegos um pouco antes de sua saída e independência dos ninhos, além de serem encontrados nos ninhos e nas imediações (Hyrenbach, 2017; Provencher et al., 2020). A coleta das fezes pode ser combinada com estudos de DNA para a determinação tanto da ingestão de resíduos poliméricos como da dieta de um indivíduo (Nelms et al., 2019).

Tabela 2 - Protocolos padronizados de coleta e armazenamento de amostras, separadas por tipo de análise, fonte e tipos de amostra.

\begin{tabular}{|c|c|}
\hline \multicolumn{2}{|c|}{$\begin{array}{c}\text { Detecção, triagem e quantificação de itens plásticos } \\
\text { Caracterização dos itens triados }\end{array}$} \\
\hline \multicolumn{2}{|l|}{ Aves vivas - conteúdo gastrointestinal } \\
\hline $\begin{array}{l}\text { Obter regurgitos inclinando a cabeça da ave e massageando } \\
\text { gentilmente o estômago e garganta e colocá-los em saco plástico } \\
\text { hermético. } \\
\text { Para análise dos itens plásticos < } 1 \mathrm{~mm} \text { coloque os regurgitos em } \\
\text { recipiente de vidro. }\end{array}$ & $\begin{array}{l}\text { - saco plástico hermético } \\
\text { - } \text { recipiente de vidro limpo, com uma folha de alumínio sob a } \\
\text { - armazenar sob congelamento para análise/transporte }\end{array}$ \\
\hline \multicolumn{2}{|l|}{ Aves mortas - conteúdo gastrointestinal } \\
\hline $\begin{array}{l}\text { Durante necropsia, extrair e amarrar as extremidades do trato } \\
\text { gastrointestinal e colocá-lo em saco plástico hermético. } \\
\text { Para análise dos itens plásticos < } 1 \mathrm{~mm} \text { envolva em uma folha dupla } \\
\text { de alumínio limpa antes de colocá-lo no saco plástico hermético. }\end{array}$ & $\begin{array}{l}\text { - bandeja de metal } \\
\text { - fórceps, pinças, tesouras limpas, lâmina de bisturi nova e } \\
\text { - linha de algodão } \\
\text { - saco plástico hermético } \\
\text { - folha dupla de alumínio nova } \\
\text { - armazenar sob congelamento para análise/transporte }\end{array}$ \\
\hline
\end{tabular}




\begin{tabular}{|c|c|}
\hline \multicolumn{2}{|l|}{ Ambiente - bolos alimentares } \\
\hline $\begin{array}{l}\text { Coletar os bolos alimentares com a pinça e colocá-los } \\
\text { individualmente em sacos plásticos herméticos. } \\
\text { Para análise dos itens plásticos < 1mm envolva em uma folha dupla } \\
\text { de alumínio limpa antes de colocá-lo no saco plástico hermético. }\end{array}$ & $\begin{array}{l}\text { - pinça (ou fórceps) limpa } \\
\text { - sacos plásticos herméticos } \\
\text { - folha dupla de alumínio limpa } \\
\text { em um local seco e escuro até o momento da análise }\end{array}$ \\
\hline \multicolumn{2}{|l|}{ Ambiente - fezes } \\
\hline Coletar fezes em tubos de tampa de rosca. & $\begin{array}{l}\text { - espátulas de madeira descartáveis } \\
\text { - tubos de tampa de rosca } \\
\text { - armazenar sob congelamento para transporte/análise }\end{array}$ \\
\hline
\end{tabular}

\section{Análises químicas}

\section{Aves vivas - regurgitos (óleo estomacal e itens)}

Obter regurgitos inclinando a cabeça da ave e massageando gentilmente o estômago e garganta e coloque-os em recipiente de vidro.
- recipiente de vidro limpo, com uma folha de alumínio sob a tampa (ou use tampas com revestimento PTFE)

- armazenar sob congelamento para transporte/análise

\section{Aves vivas - glândula uropigial}

Massagear gentilmente a glândula na base anterior da cauda, então pressionar ligeiramente até que verta óleo (uma gota).

Recolher o exsudato com uma espátula de aço inoxidável limpa e colocar em tubo de vidro.

Secar a glândula com filtro e guardá-lo em um envelope de folha de alumínio.
- espátula de aço inoxidável limpa e esterilizada

- tubo de vidro com folha de alumínio sob a tampa (ou tampa de rosca com revestimento PTFE)

- armazenar sob congelamento para transporte/análise

- filtro de microfibra de vidro, envelope de folha de alumínio limpa

- armazenar sob congelamento para transporte/análise

\section{Aves mortas - conteúdo gastrointestinal}

Durante necropsia, extrair e amarrar as extremidades do trato gastrointestinal, e colocar em saco plástico hermético. Embrulhar em uma folha dupla de alumínio limpa antes de colocá-lo diretamente no saco plástico hermético.

- bandeja de metal

- fórceps, pinças, tesouras limpas, lâmina de bisturi nova e esterilizada para dissecação

- linha de algodão

- saco plástico hermético

- folha dupla de alumínio limpa

- armazenar sob congelamento para análise/transporte

\section{Aves mortas - glândula uropigial}

Durante a necropsia, excisar a glândula uropigial inteira.

Embrulhar em uma folha dupla de alumínio antes de colocá-la em saco plástico hermético.
- bandeja de metal

- fórceps, pinças, tesouras limpas, lâmina de bisturi nova e esterilizada para dissecação

- folha dupla de alumínio limpa

- saco plástico hermético

- armazenar sob congelamento para transporte/ análise

\section{Aves mortas - tecido adiposo abdominal, músculo peitoral, órgãos (e.g. fígado)}

Durante a necropsia, coletar fragmentos de fígado $(>5 \mathrm{~g})$, músculo $(>3 \mathrm{~g})$ e tecido adiposo (> 2g).

Embrulhar em uma folha dupla de alumínio antes de colocar em saco plástico hermético.
- bandeja de metal

- fórceps, pinças, tesouras limpas, lâmina de bisturi nova e esterilizada para dissecação

- folha dupla de alumínio limpa

- saco plástico hermético

- armazenar sob congelamento para transporte/ análise

\section{Ambiente - Ovos não viáveis ou não eclodidos}

\section{Coletar ovos dos ninhos com luvas nitrílicas.}

Embrulhar os ovos individualmente com uma folha dupla de alumínio antes de colocar em saco plástico hermético.

- folha dupla de alumínio limpa

- saco plástico hermético

- armazenar sob congelamento para transporte/ análise

\section{Ambiente - bolos alimentares}

Coletar os bolos alimentares com pinça e envolvê-los individualmente em uma folha dupla de alumínio limpa antes de colocá-lo no saco plástico hermético.
- pinça (ou fórceps) limpa

- sacos plásticos herméticos

- folha dupla de alumínio limpa.

- armazenar sob congelamento ou secar o material e armazená-lo em um local seco e escuro até o momento da análise 


\section{Rotinas de limpeza para evitar a contaminação durante a coleta de amostras}

Medidas rígidas de limpeza de todo o material utilizado nos procedimentos devem ser adotadas, especialmente se a coleta de amostras também for destinada para a identificação $e$ triagem de itens menores que $1 \mathrm{~mm}$ e/ou para a análise química de compostos associados aos plásticos (aditivos e/ou adsorvíveis). Durante a coleta de amostras, o contato com plásticos, látex e outros (e.g. luvas, sacos, tubos, seringas, etc.) deve ser evitado. Devem ser usadas exclusivamente luvas nitrílicas para este fim.

Recomendações específicas: Lavar minuciosamente com água corrente, detergente e uma escova com cerdas naturais os materiais necessários como os tubos de vidro, bandejas de metal, bancada e utensílios reutilizáveis (e.g. pinças, tesouras, bisturis). Logo após, aplicar três enxagues de cloro (diluição 1:100) e álcool (diluição 3:1 em água destilada). Este procedimento deve ser realizado antes da coleta de amostras e entre necropsias;

1. Se houver intenção de realizar a análise química, inclua a limpeza dos tubos de vidro e utensílios reutilizáveis com os solventes: metanol (ou acetona), diclorometano (DCM) e hexano, nesta ordem, três vezes cada.

2. Todas as folhas de alumínio antes de sua utilização devem ser aquecidas a $450^{\circ} \mathrm{C}$ por seis horas em mufla (mufladas). Alguns utensílios também podem ser muflados desta forma em alternativa ao uso de solventes, desde que o material seja resistente à temperatura e tempo de forno.

3. Durante a amostragem de aves mortas, use sempre uma lâmina nova de bisturi para cada animal a fim de evitar a contaminação entre indivíduos.

4. Para evitar contaminação cruzada por partículas de plástico de outras fontes durante análises de microplásticos menores de $1 \mathrm{~mm}$, todos os materiais e instrumentos devem ser cobertos com papel alumínio muflado $\left(450^{\circ} \mathrm{C}\right.$ por seis horas) quando não estiverem em uso.

\section{Recomendações específicas para procedimentos que envolvam detecção, triagem e caracterização dos itens plásticos}

Macroplásticos: Para a triagem e análise dos macroplásticos, o conteúdo estomacal, regurgitos e bolos podem ser lavados e peneirados por uma malha de alumínio de $5 \mathrm{~mm}$ (ou $1 \mathrm{~mm}$ se for desejado identificar também os microplásticos posteriormente) para facilitar a separação dos itens maiores. Os itens recuperados devem ser secos à temperatura ambiente e armazenados até a análise visual. Boas práticas para categorização física (tamanho, cor, tipo, etc.) dos itens $e$ apresentação dos resultados estão detalhadas em Provencher et al. (2017) e GESAMP (2019). Ainda, a caracterização química dos itens triados por espectroscopia e particularmente útil para verificar se os itens suspeitos são polímeros sintéticos, para determinar a composição geral dos itens triados, ou ambos (FTIR associada à Reflexão Total Atenuada ATR: Álvarez et al., 2018; Lusher et al., 2018; Nelms et al., 2019; Avery-Gomm, 2020).

Microplásticos: A análise dos microplásticos requer etapas adicionais de pré-tratamento das amostras. A seguir, fornecemos um guia geral para a detecção, triagem, quantificação $e$ caracterização dos microplásticos:

Passo 1: remoção do material inorgânico de amostra: pode ser usada uma solução de $5 \mathrm{~mol} / \mathrm{L}$ de $\mathrm{NaCl}$ (flutuação ou sedimentação fracionada) para separar areia e outros materiais inorgânicos dos itens plásticos (Provencher et al., 2019). Caso as amostras não sejam úmidas, podem ser posicionadas por uma a duas horas em recipiente com água destilada pré-filtrada a fim de hidratá-las antes do processamento.

Passo 2: remoção do material orgânico de amostra: proceder com a digestão enzimática (e.g. Proteinase $\mathrm{K}$, Lipase), ácida (e.g. $\mathrm{HNO}_{3}$, $\mathrm{HClO}_{4}, \mathrm{CH}_{2} \mathrm{O}_{2}$ ), alcalina (e.g. $\mathrm{KOH}$ e $\mathrm{NaOH}$ ) ou oxidação (e.g. $\mathrm{H}_{2} \mathrm{O}_{2}$ ) (Cole et al., 2014; Lusher et al., 2017; Provencher et al., 2018b; Lavers et al., 2019). Para garantir a decomposição completa recomendamos incluir a incubação em forno de secagem a $40^{\circ} \mathrm{C}$. (Nota: este passo deve ser utilizado para itens $<1 \mathrm{~mm}$ e/ou amostras com elevado conteúdo de matéria orgânica). Considerar que dependendo do tipo de digestão utilizada, a integridade do plástico 
presente nas amostras pode ser afetada (e.g. alterações de coloração e massa), conforme especificado por Lusher et al. (2017).

Passo 3: triagem dos itens plásticos (filtrado de amostras): a malha utilizada determina o tamanho mínimo que é desejado para a amostragem. Peneiras de um milímetro são comumente utilizadas e recomendadas para o diagnóstico de microplásticos (1-5mm). Para partículas menores $(<1 \mathrm{~mm})$ é recomendada uma segunda filtração à vácuo usando equipamentos como um funil de vidro de Büchner com um filtro. Cada laboratório pode adotar diferentes tipos de filtro que serão necessários no caso da caracterização de polímeros por espectroscopia, dependendo dos equipamentos disponíveis. Posteriormente, os itens triados de forma individual e/ou em filtros devem ser colocados em placa de Petri lavada com água destilada pré-filtrada cobertos por uma folha de alumínio e mantidas por pelo menos $24 \mathrm{~h}$ à temperatura ambiente ou no mínimo $12 \mathrm{~h}$ em um forno de secagem a $40^{\circ} \mathrm{C}$.

Passo 4: identificação visual (detecção), quantificação e caracterização dos itens plásticos: Para partículas de $1-5 \mathrm{~mm}$, a avaliação visual é geralmente realizada em estéreo-microscópio (Provencher et al., 2014, 2018b; Padula et al., 2020). Para facilitar sua identificação recomenda-se coloração com vermelho do Nilo, o qual faz com que as partículas de plástico se tornem fluorescentes sob luz azul (Maes et al., 2017). Após, seguir a quantificação e categorização física (tamanho, cor, tipo, etc.) (Provencher et al., 2017; GESAMP, 2019). Ainda, a caracterização química dos itens triados por meio de técnicas espectroscópicas é particularmente útil para a confirmação da análise visual dos plásticos. Caso a partícula atinja um tamanho menor a $500 \mu \mathrm{m}$, a magnificação é necessária. Para tal, há duas opções: microscopia infravermelha (IR) (500-50 $\mu \mathrm{m})$ e microscopia Raman $(50-1 \mu \mathrm{m})$ (ambas as técnicas também são citadas como microespectroscopia) (Käppler et al., 2016).

A triagem e análise de microplásticos, especialmente partículas plásticas menores que $1 \mathrm{~mm}$, requer ambiente controlado, minimizando a contaminação da amostra. Recomendações incluem trabalhar em fluxo laminar ou ambiente de ar filtrado, mantendo as amostras cobertas com papel alumínio que tenha sido preparado com aquecimento em mufla $\left(450^{\circ} \mathrm{C}\right.$ por seis horas) caso haja intervalos em sua manipulação ou processamento (Provencher et al., 2019). A equipe do laboratório deve utilizar jalecos, se possível, em cores de fácil detecção (e.g. laranja ou rosachoque) para que se detectem com facilidade as fibras oriundas das roupas utilizadas no laboratório durante o processamento das amostras.

Coleta de brancos: Recomenda-se a obtenção de controles negativos ou brancos para detectar a presença de contaminação cruzada por partículas de plástico procedente de outras fontes durante a coleta e processamento de amostras. Para esse fim, é necessário coletar triplicatas sem conteúdo amostral, que fornecerão informação sobre potenciais contaminações no ambiente durante amostragem ("branco amostral") $e$ no momento dos procedimentos laboratoriais ("branco de procedimento").

Branco amostral: Colocar um papel de filtro limpo em placa de Petri lavada com água destilada pré-filtrada e deixá-la aberta em áreas próximas à estação de amostragem.

Branco de procedimento: Processar em paralelo a um lote de amostras no laboratório um papel de filtro limpo, usando o mesmo equipamento e reagentes pela mesma duração, como se fosse uma amostra.

\section{Recomendações específicas para procedimentos que envolvam análise química de compostos associados ao plástico}

As diretrizes para coletas que visem detecção de compostos químicos são apresentadas na Tabela 2. O aprofundamento em relação aos procedimentos específicos e equipamentos utilizados para a realização das análises químicas dos compostos associados não faz parte dos objetivos deste trabalho, para maior detalhamento, consultar referências citadas na Tabela 1.

Se possível, amostras corporais (glândula uropigial, tecidos/órgãos) e itens plásticos oriundos do conteúdo estomacal devem ser coletados do mesmo animal a fim de relacionar a detecção de compostos químicos, tanto nos itens plásticos recuperados, como nas amostras corporais.

Para obter informação sobre uma possível contaminação da amostra por compostos químicos aditivos e/ou adsorvíveis, se recomenda coletar 
brancos durante a amostragem de aves vivas em campo ("branco ambiental") e/ou das aves mortas no laboratório ("branco da dissecação"). Para coletar uma amostra de branco, agite durante um minuto um tubo de vidro no ar (ou qualquer outro utensílio como uma espátula de metal ou filtro de microfibra de vidro). Coloque no vidro uma folha de alumínio aquecida $\left(450^{\circ} \mathrm{C}\right.$ por seis horas, em mufla) sob a tampa. Etiquete-o com a data, local, hora etc. e adicione a palavra "branco". Nesse caso de preparação da amostra para análises químicas, os tubos de vidro, espátulas ou filtros devem ser sempre pré-lavados conforme indicado em "Rotinas de limpeza para evitar a contaminação durante a coleta de amostras" com os solventes metanol (ou acetona), diclorometano (DCM) e hexano, nesta ordem, três vezes cada. Adicionalmente, no ambiente de processamento das amostras, também poderá ser realizado outro branco ("branco de procedimento"). Esse será processado com as outras amostras para garantir que não há contaminação durante o processamento das amostras no laboratório.

\section{Conclusão}

Mesmo que os primeiros relatos de ingestão de resíduos plásticos tenham sido publicados na década de 60 (Provencher et al., 2017; Kühn \& van Franeker, 2020), a partir da década de 2000 foi produzido um incremento significativo de trabalhos sobre a interação dos plásticos com as aves marinhas, incluindo mais de 200 espécies (Kühn et al., 2015; Kühn \& van Franeker, 2020). Há consenso de que a avaliação da exposição ao plástico nas aves marinhas requer a adoção de métodos padronizados para facilitar a comparação entre espécies e para detectar padrões espaçotemporais (van Franeker et al., 2011; AveryGomm et al., 2016; Provencher et al., 2014). A revisão apresentada no presente trabalho fornece o cenário atual das diretrizes, métodos, opções selecionadas e recomendações de padronização existentes.

Amplamente mencionada ao longo dos artigos obtidos nessa revisão, uma das principais dificuldades da aplicação de métodos de avaliação da ingestão de itens plásticos menores que $1 \mathrm{~mm} e$ exposição a contaminantes (aditivos e adsorvíveis) em Procellariiformes é a potencial contaminação das amostras, uma vez que aos mesmos podem estar presentes desde a vestimenta utilizada pelos pesquisadores aos próprios insumos laboratoriais. Além da coleta de brancos ou controles negativos, a minimização desse desafio pode ser atingida pela padronização de procedimentos e um treinamento rigoroso para o desenvolvimento das atividades de obtenção, processamento $e$ análise das amostras.

O avanço da preocupação em relação à poluição dos oceanos e seus efeitos sobre a biodiversidade é crescente e o investimento em pesquisa e avaliação dos efeitos da contaminação por plástico em animais marinhos também. Neste contexto de produção científica crescente sobre o tema, as recomendações apresentadas devem ser reavaliadas sistematicamente para incorporar tecnologias mais recentes, fáceis e baratas tão logo estejam disponíveis e validadas.

\section{Agradecimentos}

A B. D. Hardesty e B. Wienecke pelas suas contribuições aos relatórios ACAP PaCSWG4 Doc 09 e PaCSWG5 Doc 05 que apresentaram as fundações para essas diretrizes, aos membros do Grupo de Trabalho de Populações e Estado de Conservação do ACAP (PaCSWG) e aos revisores, por seus comentários e sugestões construtivas. Agradecimento adicional a todas as equipes dos laboratórios parceiros que apoiaram as análises e procedimentos testados para a redação do presente manuscrito. Este trabalho foi possível graças ao financiamento recebido pelo Acordo Internacional para a Conservação dos Albatrozes e Petréis (ACAP) - Small Grant ACAP 2018-02, que permitiu a adoção e treinamento das equipes da Argentina e Brasil nos procedimentos tratados neste manuscrito.

\section{Referências}

Acampora H, Lyashevska O, Van Franeker JA \& O'Connor I. The use of beached bird surveys for marine plastic litter monitoring in Ireland. Marine environmental research, 120: 122-129, 2016.

Álvarez G, Barros Á \& Velando A. The use of European shag pellets as indicators of microplastic fibers in the marine environment. Marine Pollution Bulletin, 137: 444-448, 2018.

Avery-Gomm $\mathrm{S}$ et al. A study of wrecked dovekies (Alle alle) in the western North Atlantic highlights the importance of using standardized methods to quantify plastic ingestion. Marine Pollution Bulletin, 113: 75-80, 2016. 
Avery-Gomm S, Provencher JF, Liboiron M, Poon FE \& Smith PA. Plastic pollution in the Labrador Sea: an assessment using the seabird northern fulmar Fulmarus glacialis as a biological monitoring species. Marine Pollution Bulletin, 127: 817-822, 2018.

Avery-Gomm S. Plastic Pollution and Conservation of Imperilled Seabird Species. Tese de Doutorado. University of Queensland. 196p., 2020.

Baak JE, Provencher JF \& Mallory ML. Plastic ingestion by four seabird species in the Canadian Arctic: Comparisons across species and time. Marine pollution bulletin, 158: 111386, 2020.

Baini $\mathrm{M}$ et al. First detection of seven phthalate esters (PAEs) as plastic tracers in superficial neustonic/ planktonic samples and cetacean blubber. Analytical Methods, 9(9): 1512-1520, 2017.

BirdLife International. The IUCN Red List of Threatened Species 2018. http://www.birdlife.org Acesso em: 15/10/2020.

Bond AL, Provencher JF, Daoust PY \& Lucas ZN. Plastic ingestion by fulmars and shearwaters at Sable Island, Nova Scotia, Canada. Marine Pollution Bulletin, 87: 68-75, 2014.

Bond AL, Hutton I \& Lavers JL. Plastics in regurgitated Flesh-footed Shearwater (Ardenna carneipes) boluses as a monitoring tool. Marine Pollution Bulletin, 168: 112428, 2021.

Bourdages MP, Provencher JF, Baak JE, Mallory ML \& Vermaire JC. 2021. Breeding seabirds as vectors of microplastics from sea to land: Evidence from colonies in Arctic Canada. Science of The Total Environment, 764: 142808, 2021.

Braune BM, Letcher RJ, Gaston AJ \& Mallory ML. Trends of polybrominated diphenyl ethers and hexabromocyclododecane in eggs of Canadian Arctic seabirds reflect changing use patterns. Environmental Research, 142: 651-661, 2015.

Carey MJ. Intergenerational transfer of plastic debris by Short-tailed Shearwaters (Ardenna tenuirostris). Emu, 111(3): 229-234, 2011.

Codina-García M, Militão T, Moreno J \& GonzálezSolís J. Plastic debris in Mediterranean seabirds. Marine pollution bulletin, 77(1-2): 220-226, 2013.

Colabuono FI, Barquete V, Domingues BS \& Montone RC. Plastic ingestion by Procellariiformes in southern Brazil. Marine Pollution Bulletin, 58: 93-96, 2009.

Colabuono FI, Taniguchi S \& Montone RC. Polychlorinated biphenyls and organochlorine pesticides in plastics ingested by seabirds. Marine Pollution Bulletin, 60: 630-634, 2010.
Cole M, Webb H, Lindeque PK, Fileman ES, Halsband C \& Galloway TS. Isolation of microplastics in biotarich seawater samples and marine organisms. Nature Scientific Reports, 4: 4528, 2014.

Commendatore $\mathrm{M}$ et al. Persistent organic pollutants in sediments, intertidal crabs, and the threatened Olrog's gull in a northern Patagonia salt marsh, Argentina. Marine Pollution Bulletin, 136: 533-546, 2018.

Copello S \& Quintana FR. Marine debris ingestion by Southern Giant Petrels and its potential relationships with fisheries in the Southern Atlantic Ocean. Marine Pollution Bulletin, 46: 1513-1515, 2003.

Copello S, Quintana F \& Pérez F. Diet of the southern giant petrel in Patagonia: fishery-related items and natural prey. Endangered Species Research, 6(1): 15-23, 2008.

Dias MP, Martin R, Pearmain EJ, Burfield IJ, Small C, Phillips RA, ... \& Croxall JP (2019). Threats to seabirds: a global assessment. Biological Conservation, 237: 525-537.

Endo $\mathrm{S}$ et al. Concentration of polychlorinated biphenyls (PCBs) in beached resin pellets: Variability among individual particles and regional differences. Marine Pollution Bulletin, 50: 1103-1114, 2005.

Finkelstein $\mathrm{ME}$ et al. Contaminant-associated alteration of immune function in black-footed albatross (Phoebastria nigripes), a North Pacific predator. Environmental Toxicology and Chemistry: An International Journal, 26: 1896-1903, 2007.

Fossi MC et al. Are baleen whales exposed to the threat of microplastics? A case study of the Mediterranean fin whale (Balaenoptera physalus). Marine Pollution Bulletin, 64(11): 2374-2379, 2012.

Fossi MC et al. Large filter feeding marine organisms as indicators of microplastic in the pelagic environment: the case studies of the Mediterranean basking shark (Cetorhinus maximus) and fin whale (Balaenoptera physalus). Marine Environmental Research, 100: 17-24, 2014.

Fossi MC, Vlachogianni T, Galgani F, Degli Innocenti F, Zampetti G \& Leone G. Assessing and mitigating the harmful effects of plastic pollution: the collective multistakeholder driven Euro-Mediterranean response. Ocean \& Coastal Management, 184: 105005, 2020.

Furness RW. Plastic particle pollution: accumulation by procellariiform seabirds at Scottish Colonies. Marine Pollution Bulletin, 16(3): 103-106, 1985.

GESAMP. Guidelines for the monitoring and assessment of plastic litter and microplastics in the ocean. In: Kershaw PJ, Turra A \& Galgani F (Eds.), (IMO/FAO/ UNESCO-IOC/UNIDO/WMO/IAEA/UN/UNEP/UNDP/ 
ISA Joint Group of Experts on the Scientific Aspects of Marine Environmental Protection (GESAMP). Rep. Stud. GESAMP, 99: 130, 2019.

Gil-Delgado JA, Guijarro D, Gosálvez RU, LópezIborra GM, Ponz A \& Velasco A. Presence of plastic particles in waterbirds faeces collected in Spanish lakes. Environmental Pollution, 220: 732-736, 2017.

Gray H, Lattin GL \& Moore CJ. Incidence, mass and variety of plastics ingested by Laysan (Phoebastria immutabilis) and Black-footed Albatrosses (P. nigripes) recovered as by-catch in the North Pacific Ocean. Marine pollution bulletin, 64(10): 2190-2192, 2012.

Grimaldi WW, Seddon PJ, Lyver POB, Nakagawa $S$ \& Tompkins DM. Infectious diseases of Antarctic penguins: current status and future threats. Polar Biology, 38: 591-606, 2015.

Hamilton BM et al. Microplastics around an Arctic seabird colony: Particle community composition varies across environmental matrices. Science of The Total Environment, 773: 145536, 2021.

Hammer S, Nager RG, Johnson PCD, Furness RW \& Provencher JF. Plastic debris in great skua (Stercorarius skua) pellets corresponds to seabird prey species. Marine Pollution Bulletin, 103(1-2): 206-210, 2016.

Hardesty BD, Holdsworth D, Revill AT \& Wilcox C. A biochemical approach for identifying plastics exposure in live wildlife. Methods in Ecology and Evolution, 6: 92-98, 2015.

Herzke $\mathrm{D}$ et al. Negligible impact of ingested microplastics on tissue concentrations of persistent organic pollutants in northern fulmars off coastal Norway. Environmental science \& technology, 50(4): 1924-1933, 2016.

Hyrenbach KD et al. Plastic ingestion by Black-footed Albatross Phoebastria nigripes from Kure Atoll, Hawai' i: Linking chick diet remains and parental at-sea foraging distributions. Marine Ornithology, 45: 225-236, 2017.

Ibañez AE, Morales LM, Torres DS, Borghello P, Haidr NS \& Montalti D. Plastic ingestion risk is related to the anthropogenic activity and breeding stage in an Antarctic top predator seabird species. Marine Pollution Bulletin, 157: 111351, 2020.

Jambeck JR et al. Plastic waste inputs from land into the ocean. Science, 347: 768e771, 2015.

Jaspers $\mathrm{V}$ et al. Brominated flame retardants and organochlorine pollutants in eggs of Little owls (Athene noctua) from Belgium. Environmental Pollution, 136: 81-88, 2005.

Jiménez S, Domingo A, Brazeiro A, Defeo O \& Phillips RA. Marine debris ingestion by albatrosses in the southwest Atlantic Ocean. Marine Pollution Bulletin, 96: 149-154, 2015.

Käppler A et al. Analysis of environmental microplastics by vibrational microspectroscopy: FTIR, Raman or both? Analytical and Bioanalytical Chemistry, 408: 8377-8391, 2016.

Kühn S, Bravo Rebolledo EL \& van Franeker JA. Deleterious effects of litter on marine life. In Marine anthropogenic litter. Edited by M Bergmann, L Gutow, and M Klages. Springer Open, Bremerhaven, Germany. pp. 75-116, 2015.

Kühn S \& van Franeker JA. Quantitative overview of marine debris ingested by marine megafauna. Marine pollution bulletin, 151: 110858, 2020.

Kühn S, Booth AM, Sørensen L, Van Oyen A \& Van Franeker JA. Transfer of additive chemicals from marine plastic debris to the stomach oil of northern fulmars. Frontiers in Environmental Science, 8: 138, 2020.

Kühn S. Message in a belly: Plastic pathways in Fulmars. Tese de Doutorado. Wageningen University, 2020.

Kühn S, van Oyen A, Rebolledo ELB, Ask AV \& van Franeker JA. Polymer types ingested by northern fulmars (Fulmarus glacialis) and southern hemisphere relatives. Environmental Science and Pollution Research, 28(2): 1643-1655, 2021.

Lavers JL, Bond AL \& Hutton I. Plastic ingestion by Flesh-footed Shearwaters (Puffinus carneipes): Implications for fledgling body condition and the accumulation of plastic-derived chemicals. Environmental Pollution, 187: 124-129, 2014.

Lavers JL, Stivaktakis G, Hutton I \& Bond AL. Detection of ultrafine plastics ingested by seabirds using tissue digestion. Marine Pollution Bulletin 142: 470474, 2019.

Leat $\mathrm{EH}$ et al. Influence of wintering area on persistent organic pollutants in a breeding migratory seabird. Marine Ecology Progress Series, 491: 277-293, 2013.

Le Guen C, Suaria G, Sherley RB, Ryan PG, Aliani S, Boehme L \& Brierley AS. Microplastic study reveals the presence of natural and synthetic fibres in the diet of King Penguins (Aptenodytes patagonicus) foraging from South Georgia. Environment international, 134: 105303, 2020.

Lenzi J, Burgues MF, Carrizo D, Machín E \& Teixeirade Mello F. Plastic ingestion by a generalist seabird on the coast of Uruguay. Marine pollution bulletin, 107(1): 71-76, 2016.

Limonta $G$ et al. Microplastics induce transcriptional changes, immune response and behavioral alterations in adult zebrafish. Scientific reports, 9(1): 1-11, 2019. 
Lindborg VA, Ledbetter JF, Walat JM \& Moffett C. Plastic consumption and diet of Glaucous-winged Gulls (Larus glaucescens). Marine Pollution Bulletin, 64(11): 2351-2356, 2012.

Lusher AL, Hernandez-Milian G, O'Brien J, Berrow S, O'Connor I \& Officer R. Microplastic and macroplastic ingestion by a deep diving, oceanic cetacean: the True's beaked whale Mesoplodon mirus. Environmental Pollution, 199: 185-191, 2015.

Lusher AL, Welden NA, Sobral P \& Cole M. Sampling, isolating and identifying microplastics ingested by fish and invertebrates. Analytical Methods, 9(9): 13461360, 2017.

Lusher AL \& Hernandez-Milian G. Microplastic extraction from marine vertebrate digestive tracts, regurgitates and scats: a protocol for researchers from all experience levels. Bio-protocol, 8(22): e3087, 2018.

Maes T, Jessop R, Wellner N, Haupt K \& Mayes AG. A rapid-screening approach to detect and quantify microplastics based on fluorescent tagging with Nile Red. Nature Scientific Reports, 7: 44501, 2017.

Mallory ML. Marine plastic debris in northern fulmars from the Canadian high Arctic. Marine Pollution Bulletin, 56(8): 1501-1504, 2008..

Mato Y, Isobe T, Takada H, Kanehiro H, Ohtake C \& Kaminuma T. Plastic resin pellets as a transport medium for toxic chemicals in the marine environment. Environmental Science and Technology, 35: 318-324, 2001.

McInnes JC, Raymond B, Phillips RA, Jarman SN, Lea MA \& Alderman R. A review of methods used to analyse albatross diets-assessing priorities across their range. Ices Journal of Marine Science, 73(9): 21252137, 2016.

Nelms SE, Parry HE, Bennett KA, Galloway TS, Godley BJ, Santillo D : Lindeque PK. What goes in, must come out: Combining scat-based molecular diet analysis and quantification of ingested microplastics in a marine top predator. Methods in Ecology and Evolution, 10(10): 1712-1722, 2019.

Nilsen F, Hyrenbach KD, Fang J \& Jensen B. Use of indicator chemicals to characterize the plastic fragments ingested by Laysan albatross. Marine Pollution Bulletin, 87: 230-236, 2014.

Neumann S, Harju M, Herzke D, Anker-Nilssen T, Christensen-Dalsgaard S, Langset M \& Gabrielsen GW. Ingested plastics in northern fulmars (Fulmarus glacialis): A pathway for polybrominated diphenyl ether (PBDE) exposure?. Science of the Total Environment, 778: 146313, 2021.

OSPAR, 2015. Guidelines for Monitoring of Plastic Particles in Stomachs of Fulmars in the North Sea Area.
OSPAR Commission Agreement 2015-03 (Source: EIHA 15/5/12 Add.1), 26pp

Padula V, Beaudreau AH, Hagedorn B \& Causey D. Plastic-derived contaminants in Aleutian Archipelago seabirds with varied foraging strategies. Marine Pollution Bulletin, 158: 111435, 2020.

Parsons $\mathrm{M}$ et al. Seabirds as indicators of the marine environment. ICES Journal of Marine Science, 65: 1520-1526, 2008.

Perold V, Schoombie S \& Ryan PG. Decadal changes in plastic litter regurgitated by albatrosses and giant petrels at sub-Antarctic Marion Island. Marine Pollution Bulletin, 159: 111471, 2020.

Petry MV \& Benemann VR. Ingestion of marine debris by the White-chinned Petrel (Procellaria aequinoctialis): Is it increasing over time off southern Brazil?. Marine pollution bulletin, 117(1-2): 131-135, 2017.

Phillips RA et al. The conservation status and priorities for albatrosses and large petrels. Biological Conservation, 201: 169-183, 2016.

Phillips RA. Efficacy and effects of diet sampling of albatross chicks. Emu, 106: 305-308, 2006.

Phillips RA, Ridley C, Reid K, Pugh PJ, Tuck GN \& Harrison N. Ingestion of fishing gear and entanglements of seabirds: Monitoring and implications for management. Biological Conservation, 143: 501-512, 2010 .

Phillips RA \& Waluda CM. Albatrosses and petrels at South Georgia as sentinels of marine debris input from vessels in the southwest Atlantic Ocean. Environment international, 136: 105443, 2020.

Pierce KE, Harris RJ, Larned LS \& Pokras MA. Obstruction and starvation associated with plastic ingestion in a Northern Gannet Morus bassanus and a Greater Shearwater Puffinus gravis. Marine Ornithology, 32: 187-9, 2004.

Polder A, Venter B, Skaare JU \& Bouwman H. Polybrominated diphenyl ethers and HBCD in bird eggs of South Africa. Chemosphere, 73: 148-154, 2008.

Poon FE, Provencher JF, Mallory ML, Braune BM \& Smith PA. Levels of ingested debris vary across species in Canadian Arctic seabirds. Marine pollution bulletin, 116(1-2), 517-520, 2017.

Prokić MD, Radovanović TB, Gavrić JP, Faggio C. Ecotoxicological effects of microplastics: Examination of biomarkers, current state and future perspectives. TrAC Trends in analytical chemistry, 111: 37-46, 2019.

Provencher JF, Gaston AJ \& Mallory ML. Evidence for increased ingestion of plastics by northern fulmars (Fulmarus glacialis) in the Canadian Arctic. Marine Pollution Bulletin, 58: 1092-1095, 2009. 
Provencher JF et al. Prevalence of marine debris in marine birds from the North Atlantic. Marine Pollution Bulletin, 84: 411-417, 2014.

Provencher JF et al. Quantifying ingested debris in marine megafauna: a review and recommendations for standardization. Analytical Methods, 9(9): 1454-1469, 2017.

Provencher JF et al. Are ingested plastics a vector of PCB contamination in northern fulmars from coastal Newfoundland and Labrador? Environmental research, 167: 184-190, 2018a.

Provencher JF, Vermaire JC, Avery-Gomm S, Braune BM \& Mallory ML. Garbage in guano? Microplastic debris found in faecal precursors of seabirds known to ingest plastics. Science of the Total Environment, 644: 1477-1484, 2018b.

Provencher JF et al. Recommended best practices for plastic and litter ingestion studies in marine birds: Collection, processing, and reporting. Facets, 4(1): 111130, 2019.

Provencher JF et al. Are phthalate ester contaminants in northern fulmar preen oil higher in birds that have ingested more plastic? Marine Pollution Bulletin, 150: 110679, 2020.

Puskic PS, Lavers JL \& Bond AL. (2020). A critical review of harm associated with plastic ingestion on vertebrates. Science of the Total Environment, 140666.

Ríos LM, Moore C \& Jones PR. Persistent organic pollutants carried by synthetic polymers in the ocean environment. Marine Pollution Bulletin, 54: 1230-1237, 2007.

Rochman CM, Kurobe T, Flores I \& Teh SJ. Early warning signs of endocrine disruption in adult fish from the ingestion of polyethylene with and without sorbed chemical pollutants from the marine environment. Science of the Total Environment, 493: 656-661, 2014.

Roman L, Schuyler QA, Hardesty BD \& Townsend KA. Prevalence and selectivity of anthropogenic debris ingestion in eastern Australian avifauna. PLoS One, 11(8): e0158343, 2016.

Roman L, Hardesty BD, Hindell M \& Wilcox C. A quantitative analysis linking seabird mortality and marine debris ingestion. Nature Scientific Reports, 9: 1-7, 2019

Ryan PG. Seabirds indicate changes in the composition of plastic litter in the Atlantic and south-western Indian Oceans. Marine pollution bulletin, 56(8): 1406-1409, 2008.

Ryan PG, De Bruyn PN \& Bester MN. Regional differences in plastic ingestion among Southern Ocean fur seals and albatrosses. Marine Pollution Bulletin, 104: 207-210, 2016.

Savoca MS, Wohlfeil ME, Ebeler SE \& Nevitt GA. Marine plastic debris emits a keystone infochemical for olfactory foraging seabirds. Science Advances, 2: e1600395, 2016.

Sebille EV et al. The physical oceanography of the transport of floating marine debris. Environmental Research Letters, 15: 023003, 2020.

Stewart LG, Lavers JL, Grant ML, Puskic PS \& Bond AL. Seasonal ingestion of anthropogenic debris in an urban population of gulls. Marine Pollution Bulletin, 160: 111549, 2020.

Tanaka K, Takada H, Yamashita R, Mizukawa K, Fukuwaka M \& Watanuki Y. Accumulation of plastic derived chemicals in tissues of seabirds ingesting marine plastics. Marine Pollution Bulletin, 69: 219-222, 2013.

Tanaka K, Takada H, Yamashita R, Mizukawa K, Fukuwaka M \& Watanuki Y. Facilitated leaching of additive-derived PBDES from plastic by seabirds' stomach oil and accumulation tissues. Environmental Science \& Technology, 49: 11799-11807, 2015.

Tanaka K, Yamashita R \& Takada H. Transfer of hazardous chemicals from ingested plastics to highertrophic-level organisms. In Hazardous Chemicals Associated with Plastics in the Marine Environment. Springer, Cham, 267-280, 2018.

Tanaka K, van Franeker JA, Deguchi T \& Takada H. Piece-by-piece analysis of additives and manufacturing byproducts in plastics ingested by seabirds: Implication for risk of exposure to seabirds. Marine pollution bulletin, 145: 36-41, 2019.

Tanaka K, Watanuki Y, Takada H, Ishizuka M, Yamashita R, Kazama M \& Nakayama SM. (2020). In vivo accumulation of plastic-derived chemicals into seabird tissues. Current Biology, 30(4): 723-728.

Tavares D, de Moura J, Merico A \& Siciliano S. Incidence of marine debris in seabirds feeding at different water depths. Marine Pollution Bulletin, 119: 68-73, 2017.

Titmus AJ \& Hyrenbach KD. Habitat associations of floating debris and marine birds in the North East Pacific Ocean at coarse and meso spatial scales. Marine Pollution Bulletin, 62: 2496-2506, 2011.

Tourinho PS, do Sul JAI \& Fillmann G. Is marine debris ingestion still a problem for the coastal marine biota of southern Brazil? Marine Pollution Bulletin, 60(3): 396-401, 2010.

Uhart M, Gallo L \& Serafini PP. Sampling guidelines to assess plastic ingestion in ACAP species. Agreement on the Conservation of Albatrosses and Petrels. 21 p., https:// 
www.acap.aq/all-the-docs/english/acap-conservationguidelines/3728-plastics-sampling-guidelines/file, 2020.

van Franeker $\mathrm{J}$ et al. Monitoring plastic ingestion by the northern fulmar Fulmarus glacialis in the North Sea. Environmental Pollution, 159: 2609e2615, 2011.

Weimerskirch H, Inchausti P, Guinet C \& Barbraud C. Trends in birds and seals population as indicators of a system shift in the Southern Ocean. Antarctic Science, 15: 249-256, 2003.

Wilcox C, Van Sebille E \& Hardesty BD. Threat of plastic pollution to seabirds is global, pervasive, and increasing. Proceedings of the National Academy of Sciences, 112: 11899-11904, 2015.
Yamashita R, Takada H, Fukuwaka MA \& Watanuki Y. Physical and chemical effects of ingested plastic debris on short-tailed shearwaters, Puffinus tenuirostris, in the North Pacific Ocean. Marine pollution bulletin, 62(12): 2845-2849, 2011.

Yorio P, Marinao C, Kasinsky T, Ibarra C \& Suárez N. Patterns of plastic ingestion in kelp gull (Larus dominicanus) populations breeding in northern Patagonia, Argentina. Marine Pollution Bulletin, 156, 111240, 2020.

\section{Biodiversidade Brasileira - BioBrasil. \\ Fluxo Contínuo}

$$
\text { n. 3, } 2021
$$

http://www.icmbio.gov.br/revistaeletronica/index.php/BioBR

Biodiversidade Brasileira é uma publicação eletrônica científica do Instituto Chico Mendes de

Conservação da Biodiversidade (ICMBio) que tem como objetivo fomentar a discussão e a disseminação de experiências em conservação e manejo, com foco em unidades de conservação e espécies ameaçadas.

ISSN: 2236-2886 